\title{
Acquired Chiari malformation following spinal cord injury-a case series
}

\author{
David McKean ${ }^{1}$ - Umme Sara Zishan ${ }^{2}$ - Sarah Billingsley ${ }^{1}$ - Shyam S. Swarna $\mathbb{1}^{1}$ - Cormac O'Neill ${ }^{1}$. \\ Monika Banerjee $^{3} \cdot$ Safa Siddiqi $^{1} \cdot$ Joseph Papanikitas ${ }^{1} \cdot$ Sarah Yanny $^{1} \cdot$ Richard Hughes $^{1} \cdot$ Tom Meagher $^{1}$
}

Received: 6 November 2018 / Revised: 24 June 2019 / Accepted: 26 June 2019

(c) International Spinal Cord Society 2019

\begin{abstract}
Introduction Chiari malformation is characterized by caudal descent of the cerebellar tonsils through the foramen magnum. Acquired Chiari malformations (ACM) have previously been described after a variety of pathologies including lumbar puncture, cerebrospinal fluid (CSF) drainage, lumboperitoneal shunts, and conditions causing craniocephalic disproportion. Case presentation We present four cases of ACM following spinal cord injury (SCI), which has not previously been described in the literature.

Discussion ACM is rare and typically associated with abnormalities in CSF pressure or space-occupying lesions. This case series describes the potential association of SCI with ACM. We discuss the imaging findings and clinical management of these patients. Early recognition and intervention may be important to prevent progressive neurology in this vulnerable patient group.
\end{abstract}

\section{Introduction}

The development of cerebellar tonsillar descent, also referred to as an acquired Chiari malformation (ACM), has been described as a recognized but rare complication following lumboperitoneal shunting, spontaneous cerebrospinal fluid (CSF) leakage, and other conditions, which result in craniocephalic disproportion including pseudotumour cerebrii and craniosynostosis [1-3]. In addition, space-occupying lesions such as meningiomas, medulloblastomas, epidermoid cysts, and arachnoid cysts have been reported in the literature as the rare causes of ACM and syringomyelia. The pathogenesis of ACM is not well understood but the predominant theory suggests that it is related to abnormal craniospinal pressure gradients [4]. Spinal cord injury (SCI) following spinal trauma is known to

David McKean

david.mckean@nhs.net

1 Stoke Mandeville Hospital, Buckinghamshire Healthcare NHS Trust, Aylesbury, UK

2 Queen Elizabeth University Hospital, NHS Greater Glasgow and Clyde, Glasgow, UK

3 Oxford University Hospitals NHS Trust, Oxford, UK cause disturbances in spinal anatomy and abnormal CSF flow dynamics [5]. We report four cases of acquired tonsillar descent, which developed in patients with a history of SCI.

\section{Case presentation}

\section{Case 1}

A 3-year 4-month-old male child suffered an incomplete tetraplegia following a road traffic accident as a front seat passenger. Injuries at the time of presentation included diffuse axonal injury and complete $\mathrm{C} 1 / \mathrm{C} 2$ dislocation (Fig. 1a.). Additional injuries included right radial and ulnar fractures and a right median nerve neuropraxia. The patient was treated for several weeks with halo stabilization.

Routine follow-up MRI of the spine at 8 months post SCI demonstrated that the patient had an ACM with tonsillar herniation of $\sim 13 \mathrm{~mm}$ into the vertebral canal (Fig. 1b). There was no relevant clinical history of lumboperitoneal shunting or evidence of an intracranial space-occupying lesion. He had no significant change in his neurological status and was managed conservatively. He had annual MR imaging and clinical assessment showing no radiological or clinical deterioration. He continued to mobilize with an unsteady gait using a Kaye walker and no significant 
a

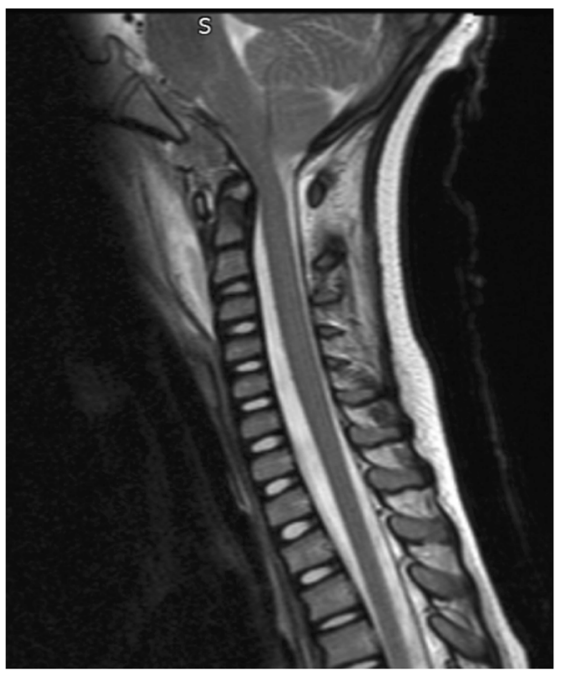

Fig. 1 a Sagittal T2 image of the cervical spine. There is evidence of traumatic occipito-cervical dissociation with significant widening of the basion-dens interval and haematoma at the expected location of the anterior atlanto-occipital ligament. Widening of the condyle-to-C1 interval and C1-C2 interspinous ligament disruption is also observed. A large hyperintense prevertebral haematoma extends from the clivus to $\mathrm{C} 4$. Normal position of the cerebellar tonsils has been noted. b

change in his neurological function has been reported in the subsequent 18 months.

\section{Case 2}

A 1-year 10-month-old male child suffered an incomplete tetraplegia following flexion/extension SCI RTA as a rear seat passenger. Imaging at the time of presentation demonstrated cord injury at the cervicothoracic junction (Fig. 2a). He was managed conservatively with a halo device for 8 weeks.

Two years following the initial injury, the patient presented with recurrent headaches. MRI at this time demonstrated tonsillar herniation of $\sim 10 \mathrm{~mm}$ consistent with ACM. There was no relevant clinical history of lumboperitoneal shunting or evidence of an intracranial space-occupying lesion. The patient's headaches persisted and after review he had a VP shunt inserted with subsequent partial relief of symptoms. The ACM has been followed up annually with MRI and clinical assessment. The patient continues to mobilize in a wheelchair and is able to stand with support. No progressive neurological deterioration has been reported.

\section{Case 3}

A 23-year-old male presented with complete paraplegia after an RTA secondary to a spinal hematoma. He had a b

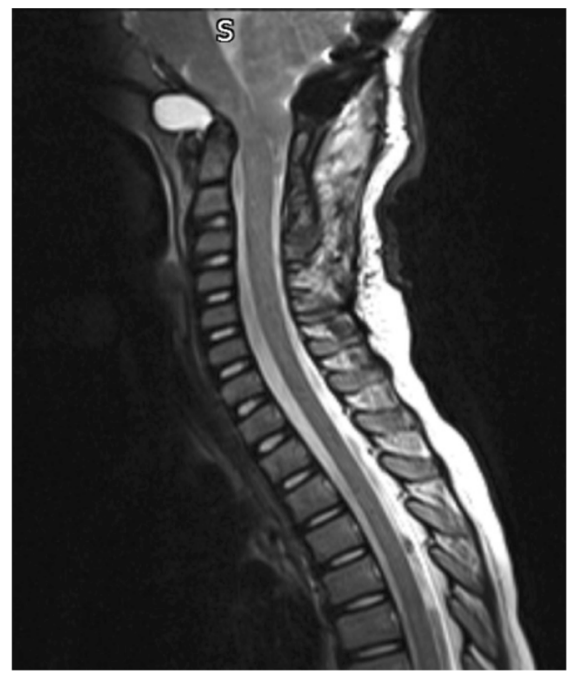

Sagittal T2 image of the cervical spine 8 months post injury. There has been interval occipitocervical fixation. There is a sizeable cyst inferior to the clivus and anterior to the apical ligament at the expected location of the anterior atlanto-occipital ligament. There is also marked descent of the cerebellar tonsils by $13 \mathrm{~mm}$, resulting in mild crowding of the brain stem and spinal cord but no focal cord signal change or syringohydromyelia

complete lesion at neurological level T2 and had been managed conservatively. Twelve years following his injury he presented with decreased function in his right hand. MRI demonstrated tonsillar descent of $12 \mathrm{~mm}$ (Fig. 3). MRI revealed extensive local dural adhesions, focal cord edema and a small central cord syrinx at the level of $\mathrm{C} 2$. There was no relevant clinical history of lumboperitoneal shunting or evidence of an intracranial space-occupying lesion. Following discussion with neurosurgery and clinical review, the decision was made to manage the patient conservatively. Annual MRI did not demonstrate any significant interval change in the extent of ACM and there has been no further progression in the patient's neurology.

\section{Case 4}

A 32-year-old male patient suffered an RTA in 2002 developing a T2 level complete paraplegia. Fifteen years following his spinal injury he presented with a new difficulty in swallowing, double vision, and loss of power in the upper limbs. MRI at this time demonstrated an ACM with tonsillar descent of $\sim 12 \mathrm{~mm}$ (Fig. 4a). There was no relevant clinical history of lumboperitoneal shunting or evidence of an intracranial space-occupying lesion. The ACM was associated with abnormal fluid intensity signal within the cervical spinal cord extending from level of $\mathrm{C} 2$ to $\mathrm{C} 4$ consistent with new syringomyelia. At T3 there was an impression of extensive local dural adhesions, a loculated 
Fig. 2 a Sagittal T2-weighted image at the time of injury. There is partial transection of the cord at the cervicothoracic junction and extensive cord oedema, with disruption of the posterior longitudinal ligament and ligamentum flavum. No Chiari malformation is present. b,c Sagittal T2- and sagittal T1weighted images 3 years post injury. Myelomalacia and posttraumatic cyst formation with focal tethering of the cord secondary to dural adhesions at the site of injury. There is tonsillar descent through the foramen magnum of $\sim 10 \mathrm{~mm}$, consistent with acquired Chiari malformation a

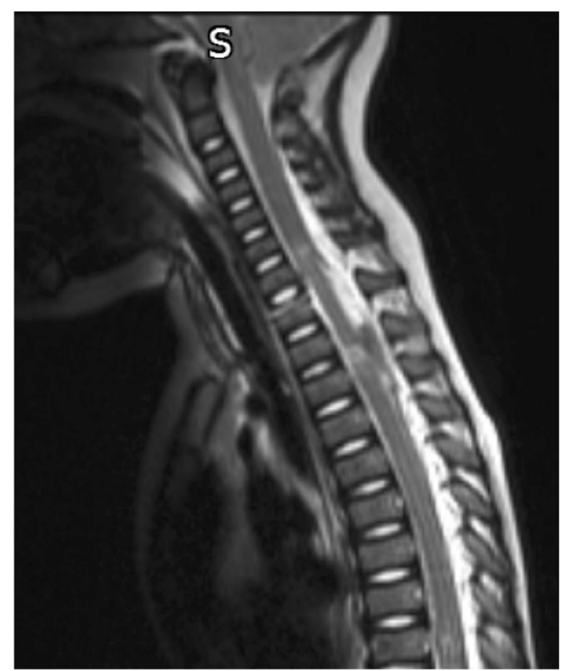

b

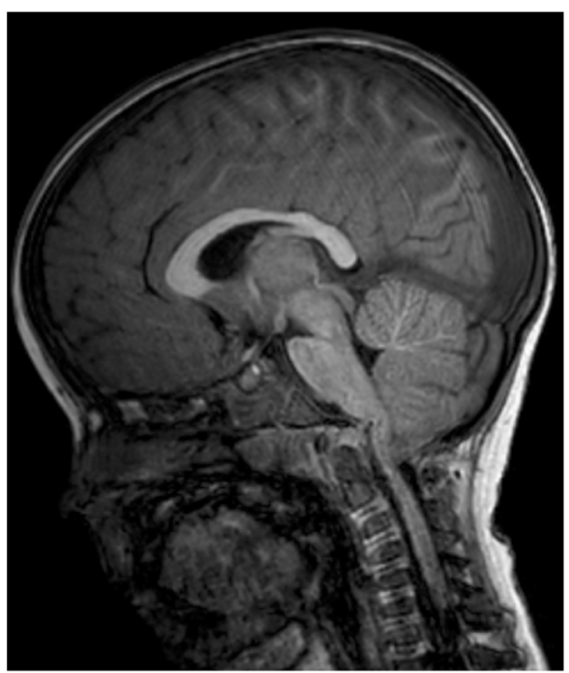

C

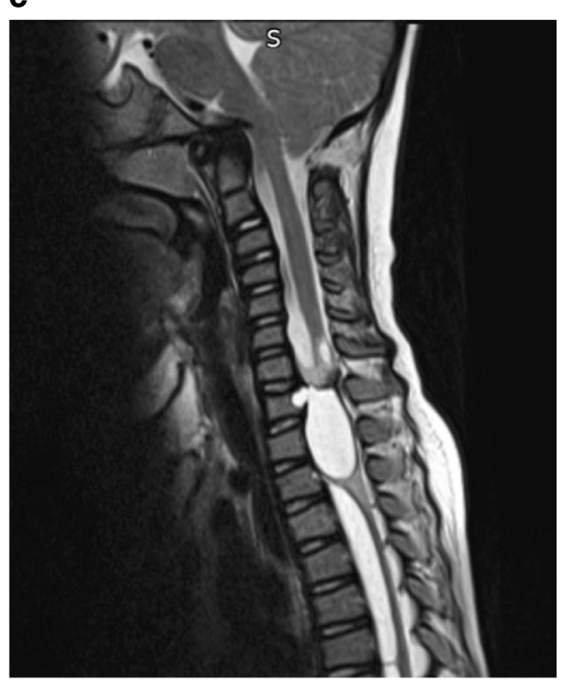

Fig. 3 Sequential T2-weighted sagittal images of the cervical spine 12 years post injury demonstrate focal myelomalacia and atrophy at the site of decompression at the $\mathrm{C} 7 / \mathrm{T} 1$ level. The cerebellar tonsils extend $10 \mathrm{~mm}$ below the level of the foramen magnum consistent with acquired Chiari

malformation. Abnormal fluid signal intensity within the spinal cord is seen at the level of $\mathrm{C} 2$ consistent with associated syringomyelia
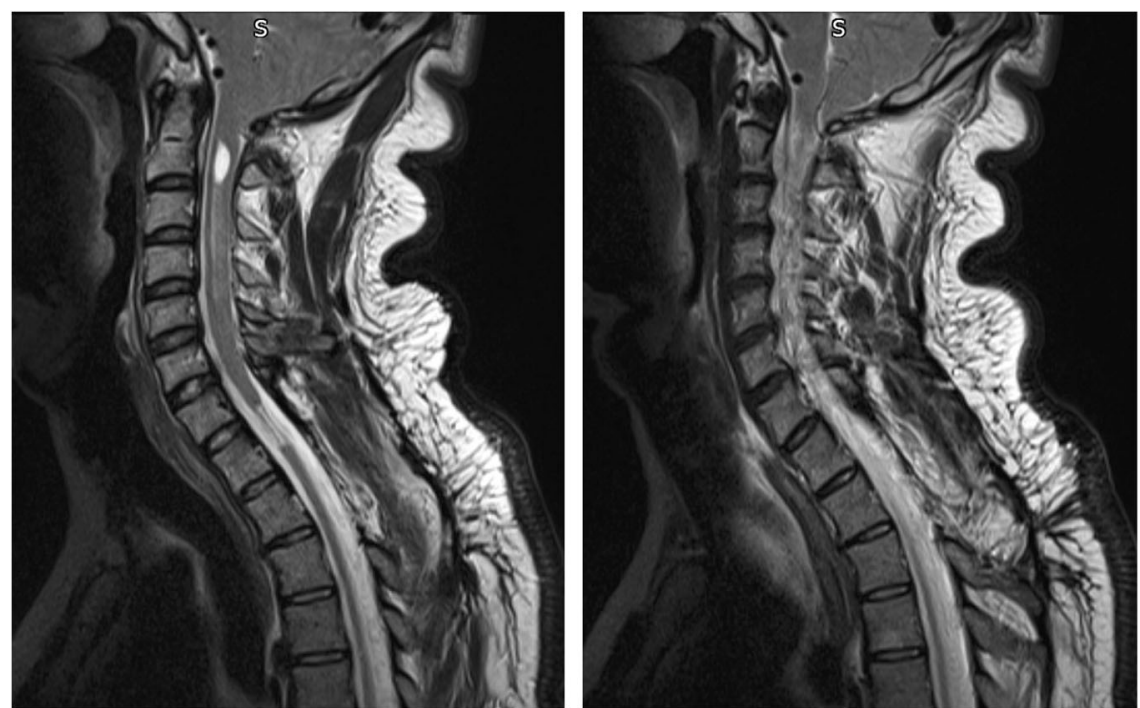
Fig. 4 a Sequential sagittal T2weighted images 15 years post injury acquired following new difficulty in swallowing, double vision and loss of upper limb power bilaterally. MRI demonstrates an acquired Chiari malformation with a tonsillar descent of $12 \mathrm{~mm}$, interstitial spinal cord oedema and mild syringomyelia extending from $\mathrm{C} 2$ to $\mathrm{C} 4$. There is evidence of previous cord injury at the $\mathrm{T} 3$ level with local dural tethering. b Cordectomy has been performed at the level of T1. There has been interval resolution of the altered cord signal cranial to the cordectomy and resolution of the acquired Chiari malformation with no significant tonsillar descent. Subcutaneous pseudomeningocele at the laminectomy site at $\mathrm{T} 2$ has been noted a
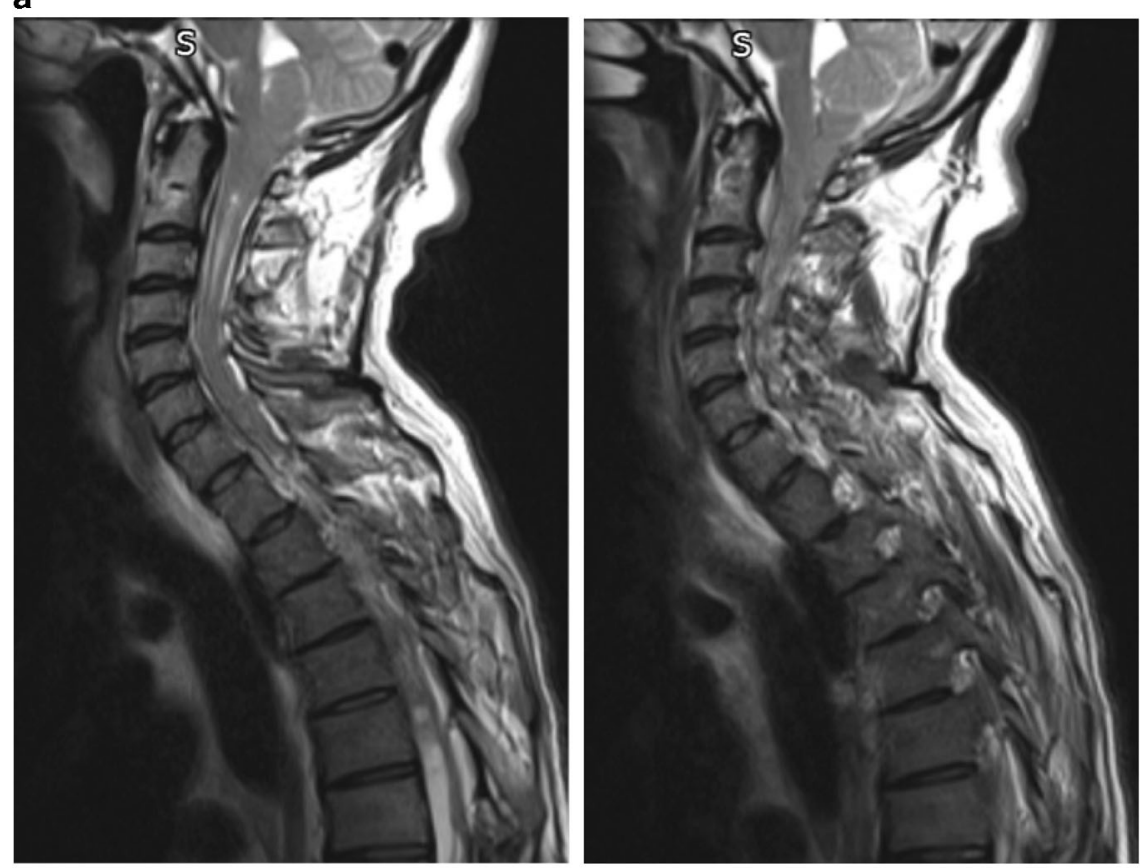

b

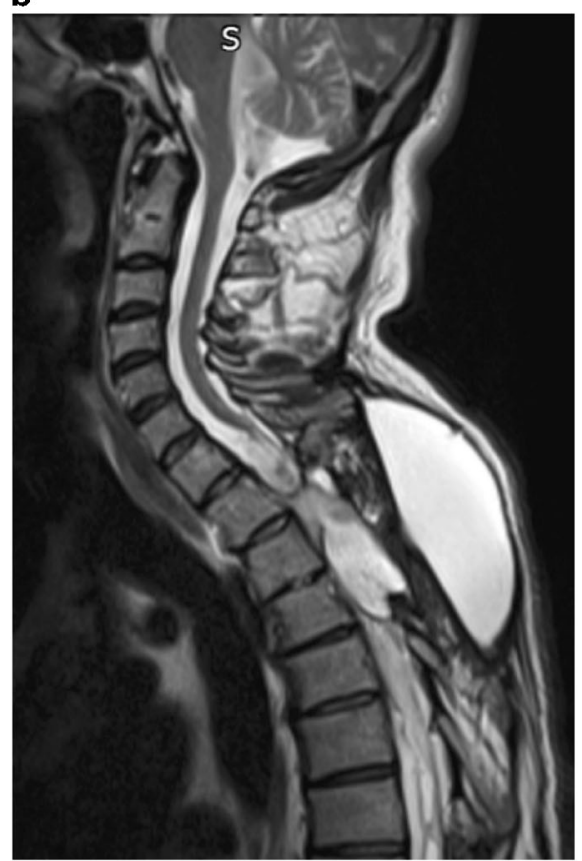

syrinx and myelomalacia of the atrophic cord at the site of prior injury.

The patient's neurological symptoms continued to deteriorate and after a mutidisciplinary discussion with the neurosurgical team the patient was put forward for operative management. He underwent a T1-T3 laminectomy and cordectomy. His new neurological symptoms resolved and he returned to his baseline of T2 complete paraplegia. No further deterioration of the patient's neurological condition was reported and follow-up MRI demonstrated resolution of the ACM.

\section{Discussion}

A Chiari malformation is a condition whereby cerebellar tissue extends into the spinal canal through the foramen magnum and can be either congenital or acquired. The most 
common type of Chiari malformation is a type 1 Chiari malformation, a structural abnormality of the posterior fossa causing cerebellar tonsillar descent through the foramen magnum. Symptoms most commonly present in adolescence or early adulthood. Type II and type III are present from birth; type II is associated with spina bifida and type III, the most severe form of ACM, is often diagnosed at prenatal ultrasound and has the highest mortality rate [6]. A number of studies have investigated the degree of cerebellar ectopia necessary to make a diagnosis of Chiari I malformation. Aboulezz et al. in 1985 reported that the average distance of the tonsillar tips was $2.9 \pm 3.4 \mathrm{~mm}$ above the foramen in 82 patients without posterior fossa abnormality, and $10.3 \pm 4.6 \mathrm{~mm}$ below the foramen in 13 patients with Chiari malformations $(p<0.005)$. They suggested that extension of the tonsils up to $3 \mathrm{~mm}$ below the foramen magnum was normal, may be considered of borderline significance between 3 and $5 \mathrm{~mm}$, and was pathologic when it exceeds $5 \mathrm{~mm}$ [7]. In their study into the radiographic findings of Chiari malformation, Milhorat et al. reported that while tonsillar herniation less than $5 \mathrm{~mm}$ does not exclude the diagnosis, the cerebellar tonsils were at least $5 \mathrm{~mm}$ below the foramen magnum in $91 \%$ of symptomatic patients in a study of 364 symptomatic patient, though there is no direct correlation between how low the tonsils are lying and clinical severity [8]. Meadows et al. reviewed the MRI images of over 22,000 patients [9], demonstrating that $0.77 \%$ of patients had cerebellar tonsillar ectopia of at least $5 \mathrm{~mm}$, however, of these patients, $14 \%$ were asymptomatic. The author emphasized that the identifying tonsillar herniation in isolation is of indeterminate clinical significance and that this must always be considered in correlation with the patients symptoms and clinical examination findings. The frequency of spinal cavitations, such as syringohydromyelia, in Chiari type 1 patients varies in the literature between 40 and $75 \%$ [8]. The prevalence of hydrocephalus associated with type 1 Chiari malformations is $~ 10 \%$ [10]. Mikulis et al. describe changing position of the cerebellar tonsils with age, with cutoffs of $6 \mathrm{~mm}$ up to 10 -year old, $5 \mathrm{~mm}$ in ages $10-30$ years, and $4 \mathrm{~mm}$ in those aged 30 years and older [11].

ACM may occur when a craniospinal pressure gradient develops naturally or iatrogenically. Conditions in which the normal cephalic absorptive pathways are disrupted whilst normal absorptive pathways in the spine are maintained may lead to a downward pressure gradient. This can occur iatrogenically secondary to lumbar punctures or CSF drainage, for example if patient has a VP shunt in situ [1214]. ACMs can also occur following spontaneous spinal CSF leakage [15]. Spontaneous CSF leakage occurs in patients who have a combination of underlying weakness of the meninges and a minor traumatic event at the cervicothoracic junction or thoracic spine. Radiologically these patients often have images mimicking a Chiari type I malformation.

The mechanism by which ACM occurs following spinal trauma is uncertain. It is possible that posttraumatic dural tethering may result in hydrodynamic changes in CSF circulation causing a downward displacement of the cerebellar tonsils. The resolution of symptomatic ACM, syrinx and cervical cord edema in case 4 of our report after the patient underwent a cordectomy lends credence to this theory.

The incidence of symptomatic ACM is reported to be rare in patients with perioperative lumbar drainage [16]. However, it is known that an ACM can cause catastrophic results including cardiopulmonary arrest or quadriplegia when it contributes to the development of acute foramen magnum syndrome. In this scenario the cerebellar tonsils acutely compress the brainstem leading to cardiovascular and neurological compromise [12]. Given that assessment of progressive neurology may be especially challenging in the context of patients with traumatic cord injury, in the case of pediatric patients or complete cervical lesions, the authors believe that awareness of the potential association of ACM following SCI is important. Awareness of this potential association may also be important for patients who require CSF lumboperitoneal shunts, when over drainage should be avoided due to the risk of brain stem compression.

\section{Conclusion}

This case series is the first to describe ACM in patients following traumatic SCI. The pathophysiology of ACM following cord injury is uncertain but may result from altered CSF circulation and a subsequent disruption of the craniospinal pressure gradient. Progressive or unacceptable neurological symptoms may necessitate intervention; as described by the authors, cordectomy resulted in the resolution of a patient's ACM, cervical cord edema and syrinx. Clinicians should be aware of the potential association between ACM and SCI as early recognition could prevent progressive neurological deterioration in this patient group.

\section{Compliance with ethical standards}

Conflict of interest The authors declare that they have no conflict of interest.

Publisher's note: Springer Nature remains neutral with regard to jurisdictional claims in published maps and institutional affiliations.

\section{References}

1. Sullivan LP, Stears JC, Ringel SP. Resolution of syringomyelia and Chiari 1 malformation by ventriculoatrial shunting in a patient 
with pseudotumour cerebri and a lumboperitoneal shunt. Neurosurgery. 1988;22:744-7.

2. Fischer EG, Welch K, Shillito J Jr. Syringomyelia following lumboureteral shunting for communicating hydrocephalus. $\mathrm{J}$ Neurosurg. 1977;47:96-100.

3. Hart A, David K, Powell M. The treatment of acquired tonsillar herniation in pseudotumour cerebri. $\mathrm{Br} \mathbf{J}$ Neurosurg. 2000;14:563-5.

4. Samii C, Möbius E, Weber W, Heienbrok HW, Berlit P. Pseudo Chiari type 1 malformation secondary to cerebrospinal fluid leakage. J Neurosurg. 1996;84:598-605.

5. Yeo J, Cheng S, Hemley S, Lee BB, Stoodley M, Bilston L. Characteristics of CSF Velocity-Time Profile in Posttraumatic Syringomyelia. AJNR Am J Neuroradiol. 2017;38:1839-44.

6. Hadley DM. The Chiari malformations. J Neuro Neurosurg Psychiatry. 2002;72:38-40.

7. Aboulezz AO, Sartor K, Geyer CA, Gado MH. Position of cerebellar tonsils in the normal population and in patients with Chiari malformation: a quantitative approach to MR imaging. J Comput Assist Tomogr. 1985;9:1033-6.

8. Milhorat TH, Chou MW, Trinidad EM, Kula RW, Mandell M, Wolpert C, et al. Chiari I malformation redefined: clinical and radiographic findings for 364 symptomatic patients. Neurosurgery. 1999;44:1005-17.
9. Meadows J, Kraut M, Guarnieri M, Haroun RI, Carson BS. Asymptomatic Chiari type I malformations identified on magnetic resonance imaging. J Neurosurg. 2000;92:920-6.

10. Nohria V, Oakes WJ. Chiari I malformation: a review of 43 patients. Pedia Neurosurg. 1990;16:222.

11. Mikulis DJ, Diaz O, Egglin TK, Sanchez R. Variance of the position of the cerebellar tonsils with age: preliminary report. Radiology. 1992;183:725-8.

12. Dagnew E, van Loveren HR, Tew JM Jr. Acute foramen magnum syndrome caused by an acquired Chiari malformation after lumbar drainage of cerebrospinal fluid: report of three cases. Neurosurgery. 2002;51:823-8.

13. Chumas PD, Armstrong DC, Drake JM, Kulkarni AV, Hoffman HJ, Humphreys RP, et al. Tonsilar herniation: the rule rather than the exception after lumboperitoneal shunting in the paediatric population. J Neurosurg. 1993;78:568-73.

14. Sathi S, Stieg PE. Acquired Chiari I malformation after multiple lumbar punctures. Neurosurgery. 1993;32:306-9.

15. Schievink WI. Spontaneous spinal cerebrospinal fluid leaks: a review. Neurosurg Focus. 2000;9:1-9.

16. Atkinson JL, Weinshenker BG, Miller GM, Piepgras DG, Mokri B. Acquired Chiari malformation secondary to spontaneous spinal cerebrospinal fluid leakage and chronic intracranial hypotension in seven cases. J Neurosurg. 1998;88:237-42. 